Inelastic cotunneling in quantum dots and molecules with weakly broken degeneracies

Begemann, Georg; Koller, Sonja; Grifoni, Milena; Paaske, Jens

Published in:

Physical Review B Condensed Matter

DOI:

10.1103/PhysRevB.82.045316

Publication date:

2010

Document version

Early version, also known as pre-print

Citation for published version (APA):

Begemann, G., Koller, S., Grifoni, M., \& Paaske, J. (2010). Inelastic cotunneling in quantum dots and molecules with weakly broken degeneracies. Physical Review B Condensed Matter, 82(4), 045316.

https://doi.org/10.1103/PhysRevB.82.045316 


\title{
Inelastic cotunneling in quantum dots and molecules with weakly broken degeneracies
}

\author{
Georg Begemann, ${ }^{1}$ Sonja Koller, ${ }^{1}$ Milena Grifoni, ${ }^{1}$ and Jens Paaske ${ }^{2}$ \\ ${ }^{1}$ Theoretische Physik, Universität Regensburg, 93040 Regensburg, Germany \\ ${ }^{2}$ The Niels Bohr Institute and Nano-Science Center, University of Copenhagen, Universitetsparken 5, DK-2100 Copenhagen $\emptyset$, Denmark
}

(Received 30 March 2010; revised manuscript received 28 June 2010; published 23 July 2010)

\begin{abstract}
We calculate the nonlinear cotunneling conductance through interacting quantum-dot systems in the deep Coulomb blockade regime using a rate equation approach based on the $T$-matrix formalism, which shows in the concerned regions very good agreement with a generalized master equation approach. Our focus is on inelastic cotunneling in systems with weakly broken degeneracies, such as complex quantum dots or molecules. We find for these systems a characteristic gate dependence of the nonequilibrium cotunneling conductance. While on one side of a Coulomb diamond the conductance decreases after the inelastic cotunneling threshold toward its saturation value, on the other side it increases monotonously even after the threshold. We show that this behavior originates from an asymmetric gate voltage dependence of the effective cotunneling amplitudes.
\end{abstract}

DOI: 10.1103/PhysRevB.82.045316

PACS number(s): 85.35.Be, 73.63.Kv

\section{INTRODUCTION}

Quantum dot devices, or so-called artificial atoms, consist of a small electronic nanostructure tunnel coupled to source and drain leads. In the Coulomb-blockade regime, sequential (one-electron) tunneling transport is exponentially suppressed and processes where two or more electrons tunnel simultaneously become the dominant transport mechanism. ${ }^{1}$ Among such correlated tunneling processes, cotunneling has received a lot of interest in recent years. Cotunneling denotes a two-electron tunneling process which can transfer an electron coherently from source to drain by a virtual population of an energetically forbidden charge state of the nanostructure. As energy is gained from the voltage drop during the electron transfer, a cotunneling event can leave the structure in an excited state, in which case one speaks of inelastic cotunneling. Otherwise the energy state of the island is left unchanged and the process is called elastic.

Inelastic cotunneling spectroscopy has turned out to be a useful tool to identify electronic, magnetic, and vibrational excitations in semiconducting ${ }^{2,3}$ or carbon-nanotube-based ${ }^{4-7}$ quantum dots as well as in single-molecule junctions. ${ }^{8-12}$ Most importantly, the positions of conductance peaks provide a very direct fingerprint of the excitation spectrum of the tunnel-coupled nanostructure but also the more detailed bias dependence or line shape of such inelastic cotunneling peaks contains valuable information. By now, it is well understood ${ }^{13-16}$ how the nonequilibrium pumping of excited states by the applied bias voltage can give rise to a cusp in the region where the bias voltage matches the relevant excitation energy. This effect is maximal for a symmetric setup. Having very different tunnel couplings to respectively source and drain electrodes implies that the nanostructure (dot or molecule) is almost equilibrated with one electrode and this effect no longer shows up. This was confirmed by an experiment by Parks et al. ${ }^{8}$ where the opening and closing of a mechanical break junction holding a $\mathrm{C}_{60}$ molecule was shown to correlate with the weakening and strengthening of such nonequilibrium cusps near the threshold for excitation of a vibrational mode in the system.

In the present paper we investigate the nonequilibrium cotunneling in a variety of complex quantum-dot systems.
For systems designed to have low-energy excitations arising from weakly broken degeneracies we find a characteristic gate dependence of the nonequilibrium modulation of the inelastic cotunneling steps. On one side of the Coulomb diamond, we find the characteristic cusped increase in conductance at threshold but on the other side of the diamond this turns into a weakening of the conductance at threshold which renders the nonlinear conductance entirely monotonous in bias. We show how this comes about by an asymmetry in the cotunneling amplitudes for processes which add or remove one electron from the dot (particle-hole asymmetry). This adds an important piece of information to the spectroscopic toolbox insofar as such a nonequilibrium depression of the cotunneling step in a symmetrically coupled device should not be mistaken for a tunnel broadened or thermally smeared cotunneling step in a very asymmetrically coupled system: such causes would lead to depression of the cotunneling steps at both sides of the Coulomb blockade diamond. In contrast, the peculiarity of our intrinsic effect is that a depression will occur only on one side while one the other the conductance will retain the typical cusped increase.

The systems we study are all coupled symmetrically to source and drain so as to maximize the nonequilibrium effects under scrutiny. They are chosen according to increasing complexity, namely, a lateral double dot (DD), a triangular triple dot (TD), and a benzene molecule. In all cases only two dots (or sites in the benzene) are tunnel coupled to the leads, see Fig. 1. For the triple dot as well as for benzene, this induces not only a breaking of the symmetry under rotations by $n \cdot 120^{\circ}$, respectively, $n \cdot 60^{\circ}(n \in \mathbb{Z})$ but also a de-

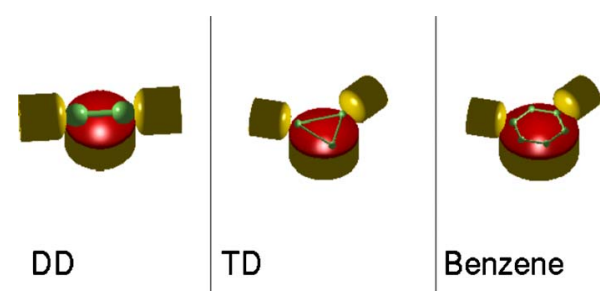

FIG. 1. (Color online) Sketch of the setups for different quantum-dot systems investigated in this work: a DD, a TD, and a "benzene" quantum dot. 
generacy lifting: while the on-site energies of all uncoupled sites can be normalized to zero, the contact sites have to be endowed with a different on-site energy $\xi$ to mimic the symmetry breaking which is likely to result from either tunneling renormalization ${ }^{7}$ or electrostatic effects. ${ }^{9,17}$

To calculate the current and other observables in transport through quantum dots in the Coulomb blockade regime there are a number of techniques, each with their advantages and limitations. Following a real-time transport approach as described in Refs. 18-21, one can trace out the leads degrees of freedom to derive a formally exact generalized master equation (GME) for the reduced density matrix of the system. This approach allows for a systematic expansion in the tunneling Hamiltonian $H_{\mathrm{T}}$, thus capturing sequential tunneling from contributions of second order in $H_{\mathrm{T}}$ and cotunneling as a fourth-order process. The GME has the advantage that it is exact to a desired order. However, already at fourth order in $H_{\mathrm{T}}$ the number of terms is quite large and therefore it is often practical to use simpler approaches which capture only the most relevant contributions for each order of the perturbation theory.

In this work, we focus entirely on the Coulomb blockade regime and we shall therefore calculate cotunneling rates within the $T$-matrix approach. ${ }^{22}$ This approach is much simpler than the GME, and will be valid deep inside a Coulomb diamond, even with the further approximations of (i) neglecting the sequential tunneling contributions and (ii) approximating the denominators in the rates as independent of the lead electron energy. To ensure that all details in the line shapes for which we aim are correct, we benchmark the above mentioned approximations as well as the $T$-matrix technique itself by a quantitative comparison to the GME approach. In general, we find the $T$-matrix and the GME approaches to be in good agreement when additional effects due to level shifts and broadening are irrelevant, namely, in the regime where the tunneling induced level broadening is much smaller than the temperature.

The paper is organized as follows: in Sec. II, we introduce the model Hamiltonian and the relevant expression to calculate the current and conductance in terms of transition rates is provided. In the end of this section, we discuss different approximations to the rates. These approximation schemes are compared against exact fourth order results in Sec. III for the case of a double-dot model. The triple dot and the benzene molecule are investigated in Sec. IV and the results are analyzed analytically using the simplest of these approximations. Conclusions are found in Sec. V

\section{MODEL}

For convenience the conventions $e=1$ and $\hbar=1$ will be used throughout the paper. A generic quantum dot coupled to source and drain leads is described by the Hamiltonian

$$
H=H_{\mathrm{QD}}+H_{\text {leads }}+H_{\mathrm{T}} \text {. }
$$

Source and drain leads are represented by two reservoirs of noninteracting electrons: $H_{\text {leads }}=\Sigma_{\alpha k \sigma}\left(\epsilon_{k}-\mu_{\alpha}\right) c_{\alpha k \sigma}^{\dagger} c_{\alpha k \sigma}$, where $\alpha=L, R$ stands for the left or right lead. The chemical potentials $\mu_{\alpha}$ of the leads depend on the applied bias voltage
$V_{\mathrm{b}}$, which is assumed to be applied symmetrically across the two junctions so that $\mu_{\mathrm{L}, \mathrm{R}}=\mu_{0} \pm \frac{V_{\mathrm{b}}}{2}$. In the following we will measure the energy starting from the equilibrium chemical potential $\mu_{0}=0$. The Hamiltonian of the quantum dot itself depends of course on the underlying nanostructure, be they quantum dots defined on semiconducting heterostructures, ${ }^{23}$ carbon nanotubes, ${ }^{24}$ or molecules bridging two contacts. ${ }^{25}$ We consider here some archetypal model for quantum dots. They can be described as two or more $(M)$ localized states coupled among each other. Following the semiempirical modeling of benzene, ${ }^{26-29}$ we study here model Hamiltonians given by

$$
\begin{aligned}
H_{\mathrm{QD}}= & \sum_{j=1}^{M} \sum_{\sigma}\left(\epsilon_{j}-\kappa V_{g}\right) d_{j \sigma}^{\dagger} d_{j \sigma}+U \sum_{j=1}^{M} n_{j \uparrow} n_{j \downarrow}+\sum_{\langle i j\rangle} b_{i j} d_{i \sigma}^{\dagger} d_{j \sigma} \\
& +\sum_{\sigma \sigma^{\prime}} \sum_{i<j} V_{i j} n_{i \sigma^{\prime}} n_{j \sigma^{\prime}}
\end{aligned}
$$

where $\epsilon_{j}$ is the on-site energy of the level $j$. In Sec. IV we will set $\epsilon_{j}=0$, except for the two sites coupled to the contacts, for which we assume $\epsilon_{j}=\xi \ll U, V_{i j}$, establishing a weakly broken degeneracy. The parameter $b_{i j}<0$ describes the hopping of electrons between nearest-neighboring states $i, j$. $U$ accounts for the on-site charging energy, while $V_{i j}$ is the interaction between two states. The first term accounts for the influence of a gate voltage $V_{g}$ with $\kappa$ being the gate coupling parameter. Its actual value strongly depends on the fabrication technique used for creating the quantum $\operatorname{dot}^{10}$ and typically ranges in order of magnitude from $10^{-3}-1$. As it simply acts as a scaling factor on the gate voltage, we can set $\kappa=1$ throughout this paper without loss of generality. The leads couple only to some of these localized states and the corresponding tunneling Hamiltonian is described by

$$
H_{\mathrm{T}}=\sum_{k \alpha \sigma}\left(t^{\alpha *} d_{j_{\alpha} \sigma}^{\dagger} c_{\alpha k \sigma}+t^{\alpha} c_{\alpha k \sigma}^{\dagger} d_{j_{\alpha} \sigma}\right)
$$

where $d_{j_{\alpha} \sigma}^{\dagger}$ creates an electron in the single particle state $|j \sigma\rangle$ which couples to lead $\alpha$. The tunneling Hamiltonian $H_{\mathrm{T}}$ is treated as a perturbation to $H_{\mathrm{QD}}+H_{\text {leads. }}$. In this work we investigate a DD, a TD, and a benzene molecule, corresponding to $M=2,3,6$ in Eq. (2), connected to source and drain as illustrated schematically in Fig. 1. We give an overview of their relevant properties in Secs. III and IV. As we shall show, weakly broken degeneracies in the TD and benzene give rise to qualitatively different inelastic cotunneling profiles at different gate voltages. This is in contrast to the DD which has a nondegenerate ground state and therefore less structure in its cotunneling amplitudes.

\section{Calculating the cotunneling current}

To make calculations easier, we shall assume $\Gamma \ll k_{B} T$ $\ll \xi \ll E_{C}$. Here, $E_{C}$ is the addition energy, which can, in principle, be expressed in terms of $U$ and $V_{i j}$, but with increasing number of sites in an increasingly unhandy way. The value of the level broadening $\Gamma$ must stay well below the thermal energy $k_{B} T$ in order to justify a perturbative approach to transport. The degeneracy lifting $\xi$ is much smaller than $E_{C}$ 
but exceeds both $\Gamma$ and the thermal energy by far. In this case, the reduced density matrix remains diagonal because one can exclude coherences between the no longer degenerate states and the rate equations are simpler. ${ }^{21,30,31}$ Moreover, normal metal leads are considered, such that a rate equation approach to transport is sufficient.

For the sequential tunneling rates, a GME approach ${ }^{18-20}$ yields the same result as Fermi's golden rule with $H_{\mathrm{T}}$ being the perturbation. The latter scheme can be iterated to include higher order tunneling processes by making use of the $T$ matrix

$$
T(E)=H_{\mathrm{T}}+H_{\mathrm{T}} \frac{1}{E-H_{0}+i 0^{+}} T
$$

from which transition rates from the initial to the final state can be calculated up to a given order in $H_{\mathrm{T}}$.

In general, the emerging $T$-matrix rates differ from the corresponding GME rates. The latter are exact to a given order in perturbation theory, and explicitly exclude all reducible terms ${ }^{32-34}$ i.e., divergences caused by the denominator in Eq. (4) going to zero. By construction the $T$ matrix misses in each order contributions guaranteeing these exclusions via a cancellation of reducible terms. Therefore unavoidable divergences emerge with the $T$-matrix technique from fourth order in the perturbation and onward. ${ }^{35,36}$ Meanwhile, regularization schemes to remove the divergence appearing in the fourth-order $T$ matrix rates have become standard ${ }^{32-34}$ and the $T$-matrix approach has been applied to various setups, e.g., to a double-dot structure ${ }^{13}$ or to molecular systems where electronic and vibronic degrees of freedom can be strongly coupled. ${ }^{32}$ In this context, it is important to stress that the standard way of regularizing the $T$-matrix rates does not exactly reproduce the GME intrinsic regularization but the discrepancy between $T$-matrix and exact perturbation theory turns out to vanish deep inside the Coulomb blockade. The same holds for further fourth-order contributions included by the GME which cannot necessarily be brought into the form of a squared matrix element ${ }^{36}$ and are disregarded by the $T$-matrix approach.

We label now the states of the quantum dot with their particle number $N$, the $S_{z}$ component of their spin with $\eta$ and an additional quantum number with $l$. The $T$-matrix rate for a transition between two states $\left|N^{\prime} l^{\prime} \eta^{\prime}\right\rangle \rightarrow|N l \eta\rangle$ of the quantum-dot system is then given by ${ }^{22}$

$$
\begin{aligned}
\Gamma_{|N l \eta\rangle\left\langle N^{\prime} l^{\prime} \eta^{\prime}\right|}= & 2 \pi \sum_{f, i} \mid\left\langle f_{N l \eta}\right| H_{\mathrm{T}} \\
& +\left.H_{\mathrm{T}} \frac{1}{E_{i_{N^{\prime} l^{\prime} \eta^{\prime}}}-H_{\mathrm{QD}}-H_{\text {leads }}+i 0^{+}} H_{\mathrm{T}}\left|i_{N^{\prime} l^{\prime} \eta^{\prime}}\right\rangle\right|^{2} \\
& \times W_{i_{N^{\prime} l^{\prime} \eta^{\prime} \eta^{\prime}}} \delta\left(E_{f_{N l \eta}}-E_{i_{N^{\prime} l^{\prime} \eta^{\prime}}}\right) .
\end{aligned}
$$

Here the sum is over all possible initial $(i)$ and final $(f)$ states of the overall system including the leads, $\left|i_{N^{\prime} l^{\prime} \eta^{\prime}}\right\rangle$ $=\left|N^{\prime} l^{\prime} \eta^{\prime}\right\rangle\left|i_{L}\right\rangle\left|i_{R}\right\rangle$, weighted by a thermal distribution function $W_{i_{N^{\prime} l^{\prime} \eta^{\prime}}}$. The rate from Eq. (5) comprises the dominant fourth-order contributions deep inside the Coulomb diamonds, namely, the cotunneling effects.
The rate equation describing the dynamics of the occupation probabilities of the states reads

$$
\dot{P}^{N l \eta}=-\sum_{N^{\prime} l^{\prime} \eta^{\prime}} \Gamma_{\left|N^{\prime} l^{\prime} \eta^{\prime}\right\rangle\langle N l \eta|} P^{N l \eta}+\sum_{N^{\prime} l^{\prime} \eta^{\prime}} \Gamma_{|N l \eta\rangle\left\langle N^{\prime} l^{\prime} \eta^{\prime}\right|} P^{N^{\prime} l^{\prime} \eta^{\prime}},
$$

where $P^{N l \eta}(t)$ is the probability of finding the dot in the state $|N l \eta\rangle$ at time $t$. The stationary solution $(t \rightarrow \infty)$ is therefore given by

$$
\sum_{N^{\prime} l^{\prime} \eta^{\prime}} \Gamma_{\left|N^{\prime} l^{\prime} \eta^{\prime}\right\rangle\langle N l \eta|} P_{\text {stat }}^{N l \eta}=\sum_{N^{\prime} l^{\prime} \eta^{\prime}} \Gamma_{|N l \eta\rangle\left\langle N^{\prime} l^{\prime} \eta^{\prime}\right|} P_{\text {stat }}^{N^{\prime} l^{\prime} \eta^{\prime}}
$$

with the normalization condition

$$
\sum_{N l \eta} P_{\text {stat }}^{N l \eta}=1
$$

With the help of the stationary solution, we arrive at an approximate expression for the current up to fourth order in $H_{\mathrm{T}}$

$$
I=I_{\text {sequential }}+I_{\text {cotunneling }}
$$

with the second order

$$
I_{\text {sequential }}=\sum_{N l \eta} \sum_{l^{\prime} \eta^{\prime}}\left(\Gamma_{\left|N+1 l^{\prime} \eta^{\prime}\right\rangle\langle N l \eta|}^{L}-\Gamma_{\left|N-1 l^{\prime} \eta^{\prime}\right\rangle\langle N l \eta|}^{L}\right) P_{\text {stat }}^{N l \eta}
$$

and fourth-order contribution

$$
I_{\text {cotunneling }}=\sum_{N l \eta}\left[\sum_{l^{\prime} \eta^{\prime}}\left(\Gamma_{\left|N l^{\prime} \eta^{\prime}\right\rangle\langle N l \eta|}^{R L}-\Gamma_{\left|N l^{\prime} \eta^{\prime}\right\rangle\langle N l \eta|}^{L R}\right)\right] P_{\text {stat }}^{N l \eta} .
$$

Here, the superscripts to the rates indicate at which lead $\alpha$ the tunneling processes take place. Truncating the general expression in Eq. (5) for the rate to second order, we retain only one tunneling event, which can either involve the left or the right lead. For the stationary current flow, we merely need to consider the balance between in- and out-tunneling at one of the electrodes, and we have chosen in Eq. (10) the left one, $\alpha=L$.

The fourth-order cotunneling events transfer an electron fully across the quantum dot, which involves two tunneling events at distinct leads. Therefore the resulting current is given by the balance between charge transfer from left to right $(R L)$ and from right to left $(L R)$. The cotunneling rates emerging from Eq. (5) can be written as

$$
\begin{aligned}
\Gamma_{|N l \eta\rangle\left\langle N l^{\prime} \eta^{\prime}\right|}^{\mathrm{eff}}= & 2 \pi \sum_{f, i}\left|\left\langle f_{N l \eta}\left|H_{\mathrm{int}}^{N}\right| i_{N l^{\prime} \eta^{\prime}}\right\rangle\right|^{2} \\
& \times W_{i_{N l^{\prime} \eta^{\prime}}} \delta\left(E_{f_{N l \eta}}-E_{i_{N l^{\prime} \eta^{\prime}}}\right),
\end{aligned}
$$

where $H_{\text {int }}^{N}$ is given by

$$
H_{\mathrm{int}}^{N}=\sum_{\alpha k \sigma} \sum_{\alpha^{\prime} k^{\prime} \sigma^{\prime}} t^{\alpha} t^{\alpha^{\prime}} h_{\eta \eta^{\prime}}^{l l^{\prime}}|N l \eta\rangle\left\langle N l^{\prime} \eta^{\prime}\right| c_{\alpha k \sigma^{\prime}}^{\dagger} c_{\alpha^{\prime} k^{\prime} \sigma^{\prime}}
$$

with matrix elements 


$$
\begin{aligned}
h_{\eta \eta^{\prime}}^{l l^{\prime}}= & {\left[\sum_{l^{\prime \prime} \eta^{\prime \prime}} \frac{\left\langle N l \eta\left|d_{j_{\alpha} \sigma}\right| N+1 l^{\prime \prime} \eta^{\prime \prime}\right\rangle\left\langle N+1 l^{\prime \prime} \eta^{\prime \prime}\left|d_{j_{\alpha^{\prime}} \sigma^{\prime}}^{\dagger}\right| N l^{\prime} \eta^{\prime}\right\rangle}{E_{N l^{\prime} \eta^{\prime}}-E_{N+1 l^{\prime \prime} \eta^{\prime \prime}}+\epsilon_{k^{\prime} \alpha^{\prime}}+i 0^{+}}\right.} \\
& \left.+\sum_{l^{\prime \prime} \eta^{\prime \prime}} \frac{\left\langle N l \eta\left|d_{j_{\alpha} \sigma}^{\dagger}\right| N-1 l^{\prime \prime} \eta^{\prime \prime}\right\rangle\left\langle N-1 l^{\prime \prime} \eta^{\prime \prime}\left|d_{j_{\alpha^{\prime}} \sigma^{\prime}}\right| N l^{\prime} \eta^{\prime}\right\rangle}{E_{N-1 l^{\prime \prime} \eta^{\prime \prime}}-E_{N l^{\prime} \eta^{\prime}}+\epsilon_{k \alpha}-i 0^{+}}\right] .
\end{aligned}
$$

Note that the effective cotunneling Hamiltonian (13) now takes the form of a generalized Kondo, or Coqblin-Schrieffer model, ${ }^{37}$ depending on the symmetries of the states $|N l \eta\rangle$.

\section{Approximation I}

To calculate the rates in Eq. (12) analytically, we neglect in a first approximation the $\epsilon_{k \alpha}$ energy dependence in the denominators of $H_{\mathrm{int}}^{N}$. This is justified for small (compared to the charging energy) bias voltages so that the electrons that tunnel to and from the leads have energies around the equilibrium chemical potential and thus $\left|E_{N \pm 1 l^{\prime \prime} \eta^{\prime \prime}}-E_{N l^{\prime} \eta^{\prime}}\right|$ $\gg\left|\epsilon_{k \alpha}\right|,\left|\epsilon_{k^{\prime} \alpha^{\prime}}\right|$. Converting the sums over $k, k^{\prime}$ into integrals assuming a flat band with constant density of states, a simple integration leads to the cotunneling rates

$$
\begin{aligned}
& \Gamma_{|N l \eta\rangle\left\langle N l^{\prime} \eta^{\prime}\right|}^{\mathrm{eff}, \mathrm{RL}}=2 \pi \sum_{\sigma \sigma^{\prime}} \nu_{L} \nu_{R}\left[-\left(E_{N l^{\prime} \eta^{\prime}}-E_{N l \eta}\right)-V_{b}\right] \\
& \times\left|\sum_{\alpha \alpha^{\prime}} \delta_{\alpha^{\prime} L} \delta_{\alpha R} t^{\alpha} t^{\alpha^{\prime}} h_{\eta \eta^{\prime}}^{l l^{\prime}}\right|^{2} n_{B}\left(E_{N l \eta}-E_{N l^{\prime} \eta^{\prime}}-V_{b}\right),
\end{aligned}
$$

where $n_{B}(x)=\frac{1}{\exp (\beta x)-1}$ is the Bose function, $\beta$ is the inverse temperature, and $\nu_{\alpha}$ is the density of states in lead $\alpha$. This approximation is valid for gate and bias voltages inside the $N$-electron Coulomb diamond. We refer to this approximation as AprxI.

\section{Approximation II}

Alternatively, to get a more precise description of the inelastic cotunneling conductance (when $\mu_{L}-\mu_{R}>E_{N l^{\prime}}-E_{N 0}$ ), we can take into account the energy dependence of $H_{\mathrm{int}}^{N}$. By shifting the integration variables $\epsilon_{k \alpha} \rightarrow \epsilon_{k \alpha}+\mu_{\alpha}$ in Eq. (14), we see that $H_{\text {int }}^{N}$ now explicitly depends on $\mu_{L}, \mu_{R}$ and therefore on the bias voltage. In the rates, we get expressions of the form

$$
\begin{aligned}
\Gamma \sim & \int d \epsilon f(\epsilon)\left[1-f\left(\epsilon+\mu_{L}-\mu_{R}+E_{N l \eta}-E_{N l^{\prime} \eta^{\prime}}\right)\right] \\
& \times \frac{1}{\epsilon-E_{1} \pm i 0^{+}} \frac{1}{\epsilon-E_{2} \pm i 0^{+}}
\end{aligned}
$$

where $E_{1}$ and $E_{2}$ depend on $l \eta$ and $l^{\prime} \eta^{\prime}$ and the summation indices in $h_{\eta \eta^{\prime}}^{l l^{\prime}}$. If $E_{1}=E_{2}$, Eq. (16) cannot be evaluated directly, because of divergences stemming from second order poles. This problem was stated already in $1994,{ }^{33}$ and a regularization scheme has been developed and become standard within the $T$-matrix approach to transport. ${ }^{32,34}$ In this regularization scheme, a finite width $\gamma \sim \Gamma$ is attributed to the states which enter the denominators as imaginary parts. This level broadening physically stems from the tunnel coupling but is not taken into account by the $T$-matrix approach. Thus the poles are shifted away from the real axis so that the integral can actually be performed. The resulting expression can be expanded in powers of $\gamma$ and the leading term is found to be of order $1 / \gamma$. Together with the prefactor of the rates, $\Gamma^{2}$, this term is identified to be a sequential tunneling term. It is excluded to avoid double counting of sequential tunneling processes. The next to leading order term is of order $\gamma^{0}$ and gives the regularized cotunneling rate. At this point, the actual value of the broadening does not matter and the limit $\gamma \rightarrow 0$ can safely be taken. The calculation of the current with regularized cotunneling processes and disregarding sequential tunneling rates $\left[I_{\text {sequential }}=0\right.$ in Eq. (9)], we refer to as AprxII.

\section{T-matrix}

Both AprxI and AprxII are expected to fail when cotunneling assisted sequential tunneling processes become accessible. This can happen well inside the Coulomb diamond, when excited $N$ particle states are populated via inelastic cotunneling. Indeed, at the lines given by the equation $\mp V_{g} \pm E_{N \pm 1 l \eta} \mp E_{N l^{\prime} \eta^{\prime}}+\mu_{\alpha}=0, l^{\prime} \neq 0$ (dashed lines inside the Coulomb diamond in Fig. 3) the cotunneling rates become negative which leads to an ill-defined set of rate equations, unless we include also sequential tunneling terms and allow also states with $N \pm 1$ to be populated. This is exactly the $T$-matrix approach, referred to as Tmat in the following.

\section{INELASTIC COTUNNELING IN A DOUBLE DOT}

In this section we discuss inelastic cotunneling features of the simplest model described by Eq. (2), namely, by a DD system. Additionally, it is used as a benchmark for the $T$-matrix approach Tmat as well as for AprxI and AprxII against a calculation based on the GME approach.

The spectrum of the DD system is shown in Fig. 2 at $V_{g}=20|b|$, corresponding to the center of the $N=2$ diamond in Figs. 3 and 4. The $N=1$ states are even and odd combinations of electron states on the left and right dot with energies $E_{\mathrm{e} / \mathrm{o}}= \pm b$. For the $N=2$ states, we have a singlet ground state and an excited triplet state, separated by $\Delta_{2}=0.5[V-U$ $\left.+\sqrt{16 b^{2}+(U-V)^{2}}\right]$. Notice the particle-hole symmetry of this system, which is responsible for the symmetry of the stability diagram around this value of the gate voltage.

In Fig. 3, we show a sketch of the stability diagram for the DD together with additional excitation lines. All the lines follow from energetical considerations involving the spectrum, see Fig. 2, and the chemical potential of the leads. We focus on the energy range relevant to the case where the dot is singly or doubly occupied, i.e., to the Coulomb diamonds with $N=1$ and $N=2$. Red lines indicate positions for transitions between states with zero and one, green lines between states with one and two, and blue lines between states with two and three electrons, respectively. Solid lines are for ground state to ground-state transitions and define the Coulomb blockade regions with $N=1$ and $N=2$, dashed lines 


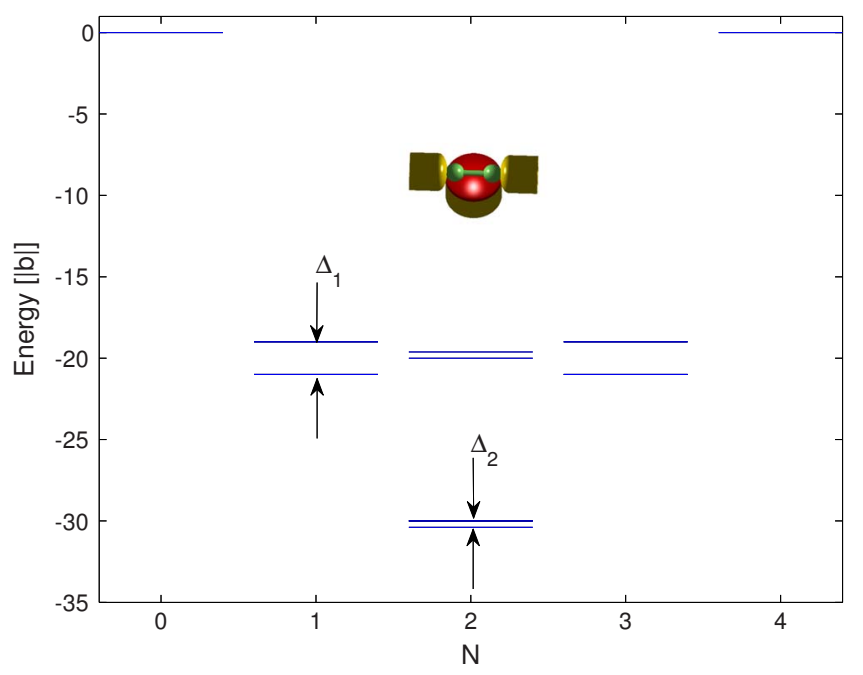

FIG. 2. (Color online) Spectrum of a double quantum dot, as described by Eq. (2) with $M=2$. The gate voltage was set to $V_{g}$ $=20|b|$, which corresponds to the center of the $N=2$ diamond (see also Figs. 3 and 4). The splitting of the $N=1$ states is $\Delta_{1}=-2 b$, the singlet-triplet splitting for the $N=2$ states is $\Delta_{2}=0.5[V-U$ $\left.+\sqrt{16 b^{2}+(U-V)^{2}}\right]$. The interaction parameters are $U=20|b|$ and $V$ $=10|b|$.

involve excited states. The dotted horizontal lines indicate the onset of the inelastic cotunneling in the two diamonds.

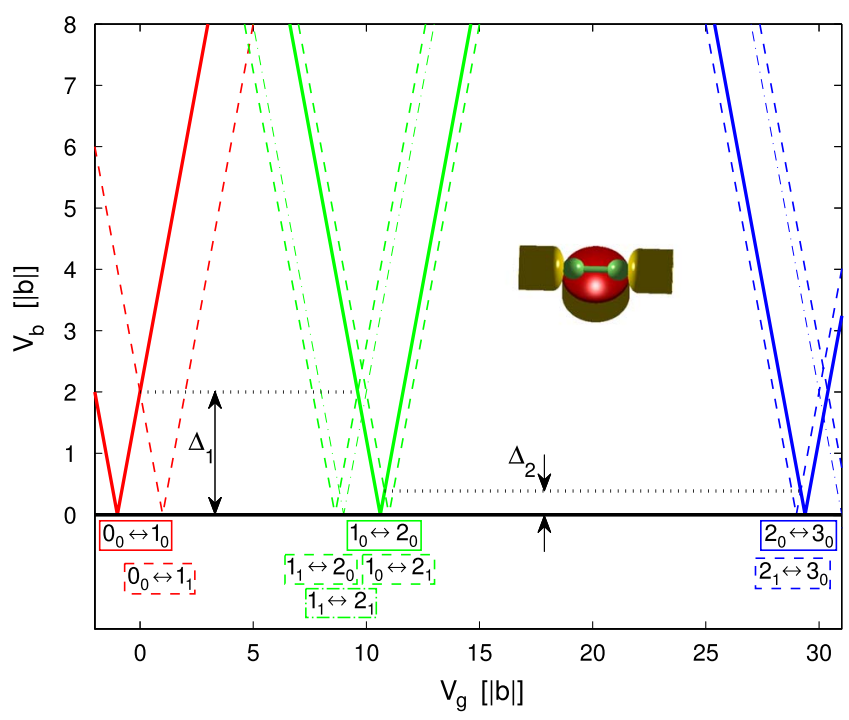

FIG. 3. (Color online) Sketch of the stability diagram for the double quantum dot described by Eq. (2) with $M=2$ and parameters $U=20|b|$ and $V=10|b|$. Red lines indicate a transition between states with zero and one, green lines between states with one and two, blue lines between two and three electrons. Solid lines are for ground state to ground-state transitions and define the Coulomb blockade regions, dashed lines involve excited states. We have labeled the participating states by $N_{i}$. It indicates the $i$ th $N$ electron state, with associated energy $E_{i}$ (for example, the one electron ground state is labeled with $1_{0}$, the first excitation with $1_{1}$, and so on). Furthermore, the dotted lines indicate the onset of the inelastic cotunneling at $V_{b}=\Delta_{1}$ in the $N=1$ and at $V_{b}=\Delta_{2}$ in the $N=2$ diamond.

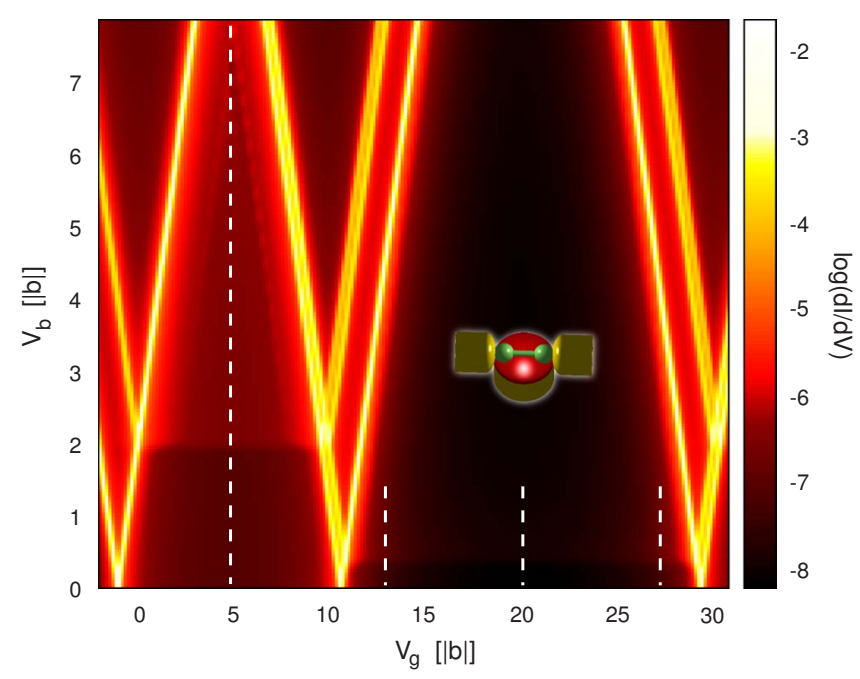

FIG. 4. (Color online) Logarithm $\left(\log _{10}\right)$ of the differential conductance $d I / d V$ for the double quantum dot as a function of gate and bias voltage calculated with the $T$-matrix approach. One recognizes the features in the $d I / d V$ discussed schematically in Fig. 3. Vertical dashed lines indicate the cuts used in Figs. 5 and 6. Parameters are as in Figs. 2 and 3, together with $k_{B} T=0.02|b|, \Gamma^{L}=\Gamma^{R}$ $=0.008|b|$. Here, as well as in all following plots, the differential conductance is measured in units of $\Phi \times e^{2} / h$, with the scaling factor $\Phi:=\Gamma^{L} \Gamma^{R} /|b|^{2}$.

In Fig. 4, the conductance through the DD calculated with Tmat is plotted on a logarithmic color scale. One can see nicely the features in the $d I / d V$ at the positions of the lines in Fig. 3 and the general resemblance of the two figures. Inside the diamonds, at $V_{b}=\Delta_{1}$ or $V_{b}=\Delta_{2}$ the threshold for inelastic cotunneling is visible as horizontal lines. The onset of the cotunneling assisted sequential tunneling (see dashed lines in Fig. 3) can be noticed best in the $N=1$ diamond. Outside the diamonds, all the sequential tunneling lines can be seen.

We are now going to compare the different approximation schemes for the cotunneling rates as discussed in the previous section with the exact perturbation theory (GME). In Fig. 5 , we show the differential conductance as a function of the bias voltage at the center of the $N=1$ diamond, as indicated by the dashed white line in Fig. 4. We see that AprxI yields good agreement with the GME only at small bias voltages. In particular, the line shape at the inelastic cotunneling threshold is not reproduced correctly, because the condition of validity for AprxI, $\Delta \ll E_{C}$, is not fulfilled here. We see that the other approaches predict an increasing differential conductance for larger bias voltages, which can be understood from the $\epsilon_{k}$ dependence in the denominators in Eq. (14).

AprxII and GME agree nicely as long as gate and bias voltages are such that one is in the innermost diamond defined by the dashed lines (see Fig. 3). Outside of this region, AprxII is no longer valid: Once the cotunneling assisted sequential tunneling sets in, the cotunneling rates in AprxII can become negative and the rate equations are ill-defined.

Inside the overall Coulomb diamond, the Tmat and GME yield almost exactly the same result. Small relative deviations (few percent) between the two approaches can be seen at the resonant lines (see inset in Fig. 5), which can be at- 


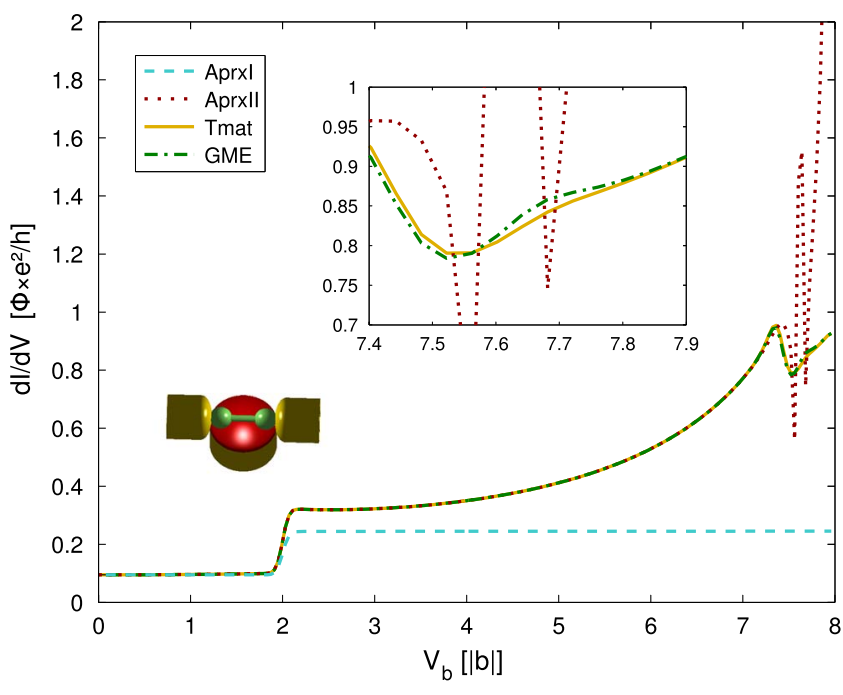

FIG. 5. (Color online) Differential conductance $d I / d V$ as a function of bias voltage calculated with the different approximation schemes discussed in Sec. II as well as with the GME at the center of the $N=1$ diamond corresponding to $V_{g}=4.8|b|$. AprxII yields divergences in the conductance at resonances. The Tmat and GME show features at these positions but are well behaved. They agree almost exactly. Parameters are as in Fig. 4.

tributed to a certain class of terms in the rates not taken into account by the $T$ matrix. ${ }^{36}$

In the $N=2$ particle diamond, a better separation of the energy scales defined by the addition energy and the inelastic cotunneling threshold is given. As expected, all approximation schemes and the GME give almost exactly the same result at the center of the diamond (see lower set of lines in Fig. 6). More toward the $N=1,2$ charge degeneracy point, at $V_{g}=13|b|$, we see that AprxI gives still a good qualitative description of the line shape of the conductance, but as in the

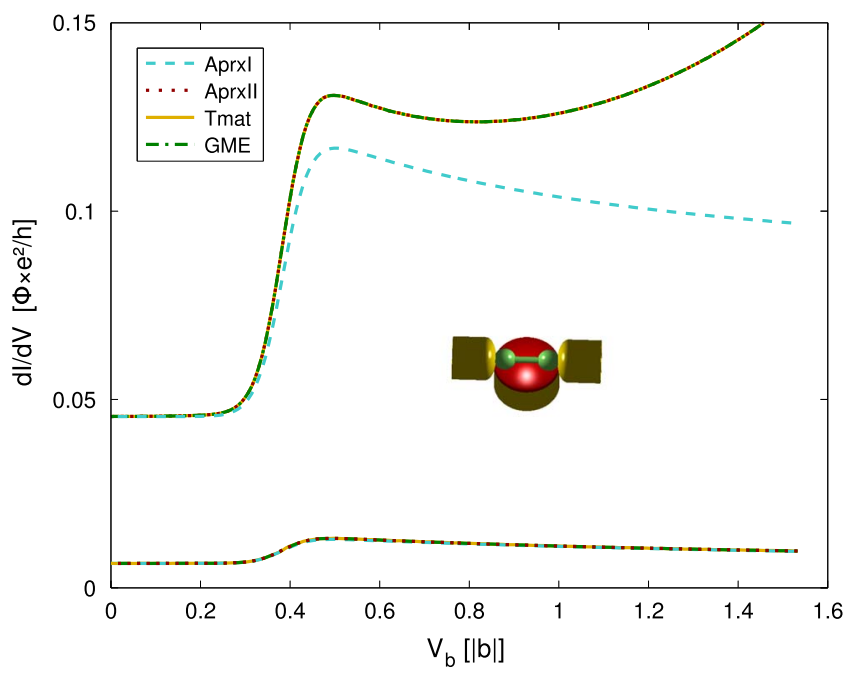

FIG. 6. (Color online) Differential conductance $d I / d V$ as a function of bias voltage calculated with different approximation schemes in the $N=2$ diamond at $V_{g}=13|b|$ (upper set of lines) and at the center of the diamond corresponding to $V_{g}=20|b|$ (lower set of lines). Due to the particle-hole symmetry of the DD, the two cuts at $V_{g}=13|b|$ and $V_{g}=27|b|$ give exactly the same result.
$N=1$ diamonds it fails to reproduce the increase in the conductance due to the bias dependence of the denominators of the rates. The decrease in the conductance after the inelastic cotunneling threshold is due to the nonequilibrium redistribution of the population of the excited state. At low bias, only the ground state is populated and only cotunneling processes that do not change the occupation of the ground state are possible. When the bias is large enough to populate the excited state, the conductance suddenly increases due to the new possibilities of transferring electrons from left to right lead. The excited state starts to acquire a finite nonequilibrium population from this point on and together with the increasing depopulation of the ground state this leads typically to a decrease in the differential conductance after the sudden increase at the threshold. We will discuss the behavior of the conductance at the inelastic cotunneling threshold in more detail in the next section. Since the DD is particlehole symmetric, cuts through the $N=2$ diamond at the same distances from the center toward the $N=1,2$ and $N=2,3$ charge degeneracy points give exactly the same result.

\section{INELASTIC COTUNNELING IN DOTS WITH WEAKLY BROKEN DEGENERACIES}

Systems with slightly broken symmetries, e.g., molecules in a single-molecule junction, exhibit weakly broken degeneracies, where the splitting of the originally degenerate states is much smaller than the addition energy. These systems provide a separation of energy scales which allows us to investigate inelastic cotunneling effects that are largely unaffected by charge fluctuations. From a technical point of view, this brings us into the validity range of the simplest approximation on the cotunneling rates (AprxI). We assume in the following a site-independent hopping $b_{i j}=b<0$ for nearest neighbors $i, j$ and a shift of the on-site energies of the contacted sites by $\xi=-0.1|b|$.

\section{A. Triangular triple dot}

A triple quantum-dot system is described by $H_{\mathrm{QD}}$ in Eq. (2) with $M=3$. We assume that the left lead is coupled to dot 1 and the right lead to dot 2 . The coupling of dots 1 and 2 to the leads breaks the symmetry of the isolated molecule, and in accordance with our previous statements we set thus $\epsilon_{2}$ $=\epsilon_{1}=\xi, \epsilon_{3}=0$. The corresponding energy spectrum is shown in Fig. 7 as a function of the number $N$ of electrons in the triple quantum dot. The gate voltage is chosen such that the lowest energy occurs when the TD is filled with $N=3$ electrons.

For $\xi=0$ the $N=3$ ground state is both spin and orbitally degenerate. We label these orbitals with $l=1,2$. For finite $\xi$, they will split by an energy of $|E(l=2)-E(l=1)|=\Delta(\xi)$, where $\Delta(\xi=-0.1|b|)$ is much smaller than the addition energy $E_{C}=U_{N=3}^{\text {add }}$ (see Fig. 7, inset). The next excited state with $N=3$ is separated by an energy comparable to the addition energy and can thus be disregarded.

In Fig. 8, we focus now on the situation when the system is filled with three electrons. For low temperatures, sequential tunneling is exponentially suppressed at small bias volt- 


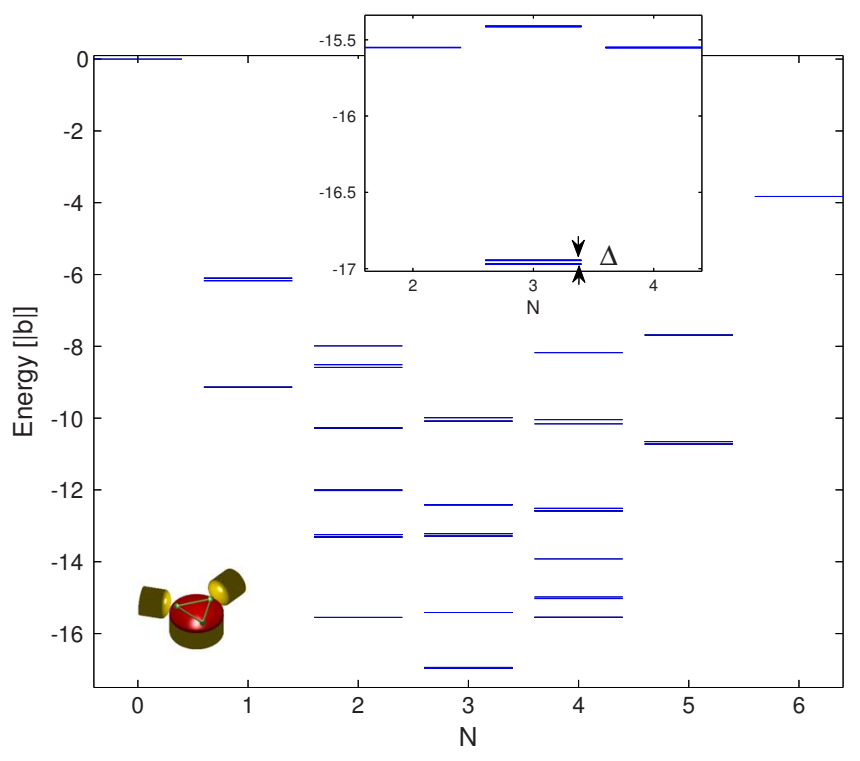

FIG. 7. (Color online) Spectrum of the triple dot at $V_{g}$ $=1.14|b|$ (center of the $N=3$ Coulomb diamond). The addition energy $E_{C}=U_{N=3}^{\text {add }}$ for $N=3$ can be read off as the distance between the $N=3$ and the $N=2$ ground state plus the distance between the $N$ $=4$ and the $N=3$ ground state. The splitting $\Delta$ of the $N=3$ ground state is about a hundred times smaller than the addition energy (see inset). Parameters are $U=5|b|, V=2|b|$, and $\xi=-0.1|b|$.

ages and the current is dominated by cotunneling events. We show the cotunneling conductance calculated with AprxI as a function of gate and bias voltage. The inelastic cotunneling threshold is clearly seen as a horizontal line at $V_{b}=\Delta$. In Fig. 9 , we show three cuts of the cotunneling conductance at

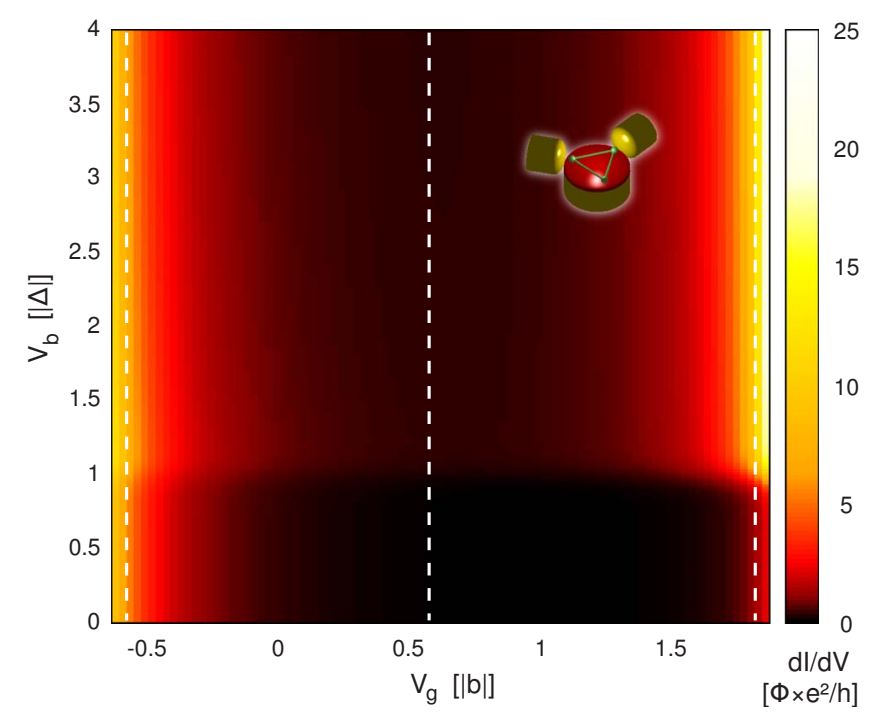

FIG. 8. (Color online) Color-coded cotunneling conductance of a TD well inside the $N=3$ Coulomb diamond. The conductance in general is lowest at the center of the diamond and increasing when moving the gate toward the diamonds with $N \pm 1$. The onset of inelastic cotunneling at $V_{b}=\Delta$ is clearly visible as a jump in the conductance. The charge degeneracy points are at $V_{g}=-0.84|b|$ (with $N=2$ ) and at $V_{g}=1.98|b|(N=4)$. Parameters as in Fig. 7, with additionally $k_{B} T=8 \times 10^{-4}|b|, \Gamma^{L}=\Gamma^{R}=8 \times 10^{-5}|b|$.

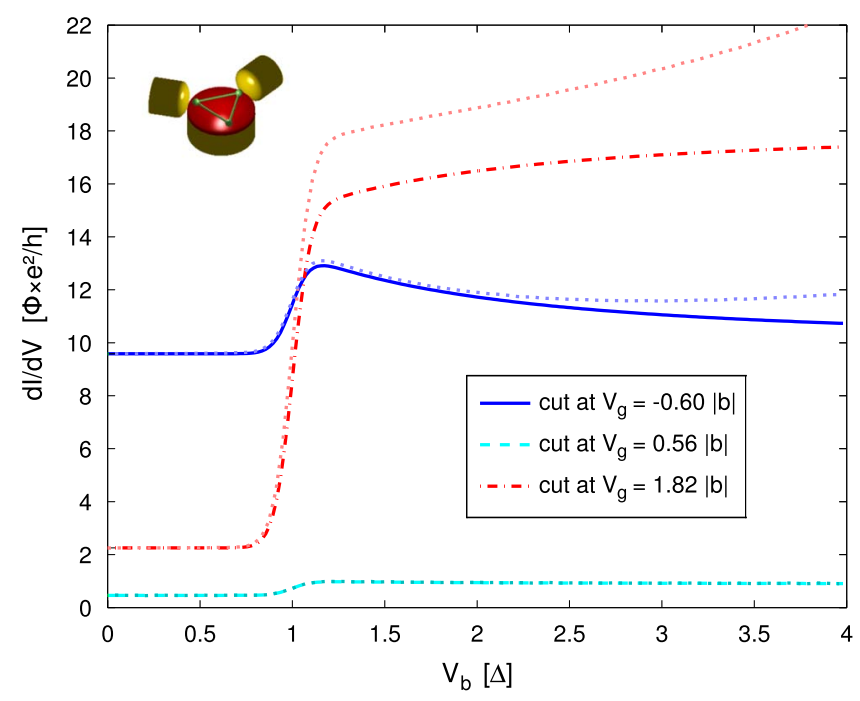

FIG. 9. (Color online) Cotunneling conductance of a TD vs bias voltage at the positions indicated by the white dashed lines in Fig. 8. The line shape as well as the magnitude depend on the gate voltage. The solid/dashed/dot-dashed curves are calculated with AprxI, their dotted companions with AprxII. The two approaches agree well for low bias voltages $V_{b} \ll U_{N=3}^{\text {add }}$.

different gate voltages (calculated with AprxI and AprxII), one at the center, two toward the corners of the $N=3$ diamond, as indicated by the dashed white lines in Fig. 8. As one can see from the comparison of the three cuts in Fig. 9, the magnitude of the conductance as well as the exact line shape now depends strongly on the gate voltage. At the center of the diamond (at $V_{g} \approx 0.56|b|$ ), and even better pronounced at lower gate voltages (e.g., at $\left.V_{g}=-0.60|b|\right)$, the conductance shows the expected behavior: it is constant below the inelastic cotunneling threshold, and above it shows a step with a cusp. The origin of this cusp lies in the nonequilibrium redistribution of the occupation probabilities of the two orbitals. For bias voltages below the threshold, only the ground state is populated. Above, the occupation probability of the excited state rises and increases with the bias, heading toward its saturation value. Until the saturation value is reached, the conductance will change with the bias. This also true for the cut at $V_{g} \approx 1.82|b|$, however, there is no cusp, but a steady further increase in conductance above the inelastic cotunneling step. To understand this different behavior, we analyze the expression for the cotunneling current and the underlying rates.

We allow only the orbitals of the split ground state to be populated. We therefore have to solve the rate Eqs. (7) and (8) for $P^{N=3 l \eta}$. For zero magnetic field, we expect $P^{N=3 l \uparrow}$ $=P^{N=3 l \downarrow}$ and conveniently we can reduce the problem to two independent variables $P_{l}=\Sigma_{\eta} P^{N=3 l \eta}, l=1,2$, where the index $N=3$ has been dropped. The following analysis is now performed under the assumptions that $T=0, V_{b}>0$, and $E(l=1)<E(l=2)$ without loss of generality.

The current is then given by

$$
I=\sum_{l l^{\prime}} \Gamma_{\left|l^{\prime}\right\rangle\langle l|}^{R L} P_{l}
$$

and the differential conductance follows as 


$$
\frac{d I}{d V_{b}}=\sum_{l l^{\prime}}\left[\Gamma_{\left|l^{\prime}\right\rangle\langle l|}^{R L} \frac{d P_{l}}{d V_{b}}+P_{l} \frac{d}{d V_{b}} \Gamma_{\left|l^{\prime}\right\rangle\langle l|}^{R L}\right] .
$$

Here, $\Gamma_{\left|l^{\prime}\right\rangle\langle l|}^{R L}$ is the cotunneling rate for changing the quantum dot from the state $l$ to $l^{\prime}$ and thereby transferring an electron from the left to the right lead. Within AprxI, we can write the total rate $\Gamma_{\left|l^{\prime}\right\rangle\langle l|}$ as in Eq. (15)

$$
\begin{aligned}
\Gamma_{\left|l^{\prime}\right\rangle\langle l|}= & \sum_{\alpha \alpha^{\prime}} \Gamma_{\left|l^{\prime}\right\rangle\langle l|}^{\alpha \alpha^{\prime}}=\sum_{\alpha \alpha^{\prime}} \gamma_{\left|l^{\prime}\right\rangle\langle l|}^{\alpha \alpha^{\prime}} \Theta\left(\mu_{\alpha^{\prime}}-\mu_{\alpha}+E_{l^{\prime}}-E_{l}\right) \\
& \times\left(\mu_{\alpha^{\prime}}-\mu_{\alpha}+E_{l^{\prime}}-E_{l}\right),
\end{aligned}
$$

where $\gamma_{\left|l^{\prime}\right\rangle\langle l|}^{\alpha \alpha^{\prime}}$ depends on the gate voltage only and the remaining terms depend only on the bias voltage. We are especially interested in the conductance slightly above the inelastic cotunneling threshold, where $V_{b}=\Delta+\epsilon, \epsilon \rightarrow 0^{+}$. At this point, still $P_{1} \approx 1$ and $P_{2} \approx 0$, while $\left.\frac{d P_{1}}{d V_{b}}\right|_{V_{b}=\Delta+\epsilon}<0,\left.\frac{d P_{2}}{d V_{b}}\right|_{V_{b}=\Delta+\epsilon}$ $>0$, and $\frac{d}{d V_{b}} \Gamma_{\left|l^{\prime}\right\rangle\langle l|}^{R L}=\gamma_{\left|l^{\prime}\right\rangle\langle l|}^{R L}$. With these inputs, we get for the conductance

$$
\begin{aligned}
\left.\frac{d I}{d V_{b}}\right|_{V_{b}=\Delta+\epsilon}= & {\left[\Gamma_{|1\rangle\langle 1|}^{R L}+\Gamma_{|2\rangle\langle 1|}^{R L}-\Gamma_{|1\rangle\langle 2|}^{R L}-\Gamma_{|2\rangle\langle 2|}^{R L} \frac{d P_{1}}{d V_{b}}\right.} \\
& +\left(\gamma_{|1\rangle<1 \mid}^{R L}+\gamma_{|2\rangle\langle 1|}^{R L}\right) P_{1}+\left(\gamma_{|1\rangle\langle 2|}^{R L}+\gamma_{|2\rangle\langle 2|}^{R L}\right) P_{2} .
\end{aligned}
$$

From this expression, one sees that if $\gamma_{|2\rangle\langle 2|}^{R L}$ is large, the contribution containing $P_{2}$, which grows with raising bias, can win, so that the conductance does not show a cusp, but increases monotonously after the step. In other words, with a sufficiently large value of $\gamma_{|2\rangle\langle 2|}^{R L}$, the conductance will keep growing once the state $l=2$ starts to be increasingly occupied at $V_{b} \gtrsim \Delta$. Below the threshold, the elastic cotunneling conductance is set by the prefactor to $P_{1}$ in Eq. (20). At very large bias, however, the two states become equally populated, the first line in Eq. (20) vanishes and the with a large value for $\gamma_{|2\rangle\langle 2|}^{R L}$, the saturation conductance can become much larger than the elastic subthreshold conductance. This implies that a monotonous increase in the conductance across the threshold will be accompanied by a large step height, i.e., a large difference between the conductance at $V_{b}=0$ and at $V_{b} \gg \Delta$. This is clearly seen to be the case in Fig. 9 .

One can make the above statements about large couplings more precise, by inserting the stationary solutions for $P_{1}$ and $P_{2}$ given by

$$
P_{1}=\frac{\Gamma_{|1\rangle\langle 2|}}{\Gamma_{|1\rangle\langle 2|}+\Gamma_{|2\rangle<1 \mid}}, \quad P_{2}=\frac{\Gamma_{|2\rangle<1 \mid}}{\Gamma_{|1\rangle\langle 2|}+\Gamma_{|2\rangle<1 \mid}}
$$

into the second derivative of the current at $V_{b}=\Delta+\epsilon$. From this, one obtains

$$
\left.\frac{d^{2} I}{d V^{2}}\right|_{V_{b}=\Delta+\epsilon}=-2 \frac{\gamma_{|2\rangle\langle 1|}^{R L}\left(\left(\gamma_{|1\rangle\langle 2|}^{R L}-\gamma_{|2\rangle\langle 1|}^{R L}\right)\left(\gamma_{|1\rangle\langle 1|}^{R L}-\gamma_{|2\rangle\langle 2|}^{R L}\right)+4 \gamma_{|2\rangle\langle 1|}^{R L} \gamma_{|1\rangle\langle 2|}^{R L}\right)}{\Delta\left(2 \gamma_{|1\rangle\langle 2|}^{R L}+\gamma_{|1\rangle\langle 2|}^{L L}+\gamma_{|1\rangle\langle 2|}^{R R}\right)^{2}}-2 \frac{\gamma_{|2\rangle\langle 1|}^{R L}\left(\gamma_{|2\rangle\langle 1|}^{R L}+\gamma_{|1\rangle<1 \mid}^{R L}-\gamma_{|1\rangle\langle 2|}^{R L}-\gamma_{|2\rangle\langle 2|}^{R L}\right)\left(\gamma_{|1\rangle\langle 2|}^{L L}+\gamma_{|1\rangle\langle 2|}^{R R}\right)}{\Delta\left(2 \gamma_{|1\rangle\langle 2|}^{R L}+\gamma_{|1\rangle\langle 2|}^{L L}+\gamma_{|1\rangle(2 \mid}^{R R}\right)^{2}},
$$

which is positive, thus giving rise to a monotonously increasing cotunneling conductance, whenever

$$
\gamma_{|2\rangle\langle 2|}^{R L}>\gamma_{|1\rangle<1 \mid}^{R L}+\frac{\left(\gamma_{|2\rangle\langle 1|}^{R L}-\gamma_{|1\rangle\langle 2|}^{R L}\right)\left(\gamma_{|1\rangle\langle 2|}^{L L}+\gamma_{|1\rangle\langle 2|}^{R R}\right)+4 \gamma_{|2\rangle\langle 1|}^{R L} \gamma_{|1\rangle\langle 2|}^{R L}}{\gamma_{|1\rangle\langle 2|}^{R L}-\gamma_{|2\rangle\langle 1|}^{R L}+\gamma_{|1\rangle\langle 2|}^{L L}+\gamma_{|1\rangle\langle 2|}^{R R}}
$$

The question now remains, under which circumstances this condition can be fulfilled. To answer this question, we have to analyze the transition amplitudes

$$
\gamma_{|l\rangle\left\langle l^{\prime}\right|}^{R L}=\sum_{\eta \eta^{\prime}}\left[\sum_{l^{\prime \prime} \eta^{\prime \prime}} \frac{\left\langle N l \eta\left|d_{j_{R} \sigma}\right| N+1 l^{\prime \prime} \eta^{\prime \prime}\right\rangle\left\langle N+1 l^{\prime \prime} \eta^{\prime \prime}\left|d_{j_{L} \sigma^{\prime}}^{\dagger}\right| N l^{\prime} \eta^{\prime}\right\rangle}{E_{N l^{\prime}}-E_{N+1 l^{\prime \prime}}}+\sum_{l^{\prime \prime} \eta^{\prime \prime}} \frac{\left\langle N l \eta\left|d_{R \sigma}^{\dagger}\right| N-1 l^{\prime \prime} \eta^{\prime \prime}\right\rangle\left\langle N-1 l^{\prime \prime} \eta^{\prime \prime}\left|d_{j_{L} \sigma^{\prime}}\right| N l^{\prime} \eta^{\prime}\right\rangle}{E_{N-1 l^{\prime \prime}}-E_{N l^{\prime}}}\right] .
$$

We see that they depend on the overlap matrix elements of the tunneling Hamiltonian in the numerator and on the energy differences of the states involved in the cotunneling process in the denominator. $\Delta$ is small compared to the addition energy, and we keep a distance to the edges of the diamonds, so that for our analysis, we can set $E^{N=3}(l=1)$ $=E^{N=3}(l=2)$ in the denominator of the above expression. However, as we approach one of the two charge degeneracy points (either $N \leftrightarrow N+1$ or $N-1 \leftrightarrow N$ ) on the axis of the gate voltage, the contributions 

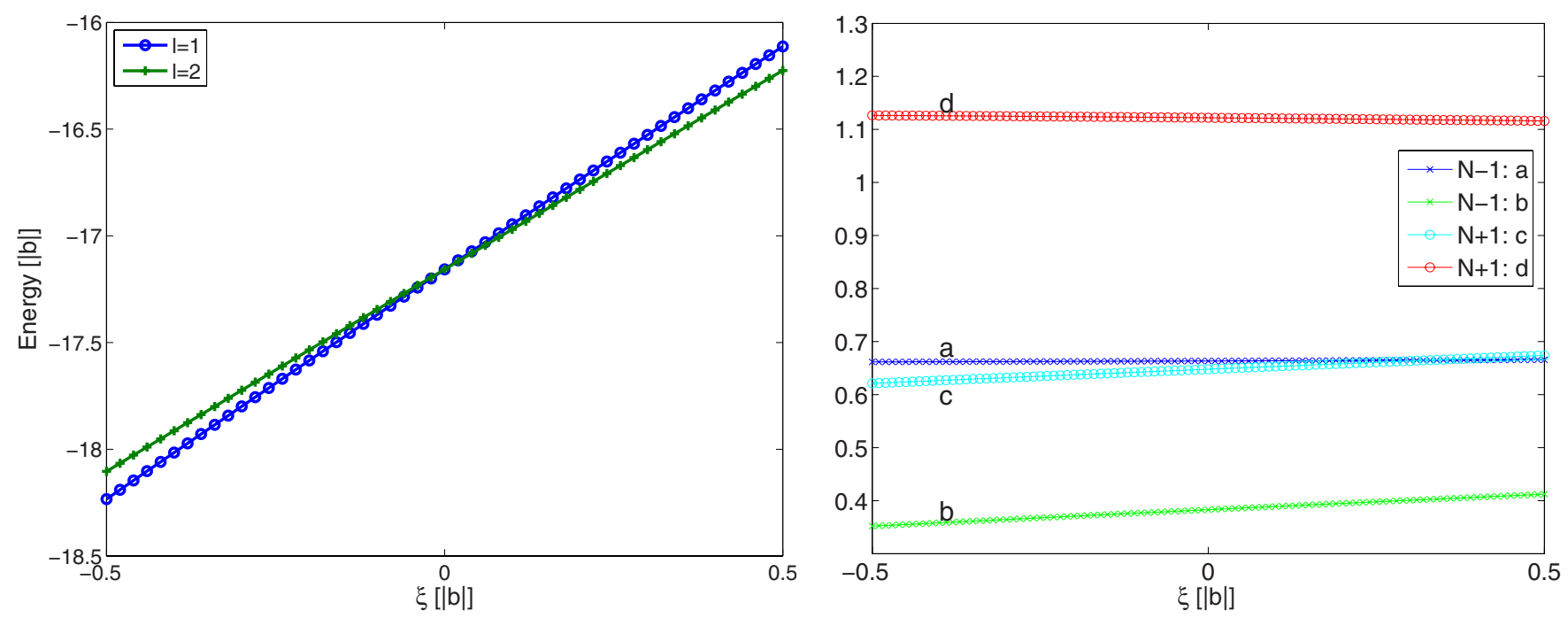

FIG. 10. (Color online) Left panel: the quasidegenerate $N=3$ ground states of a TD with $l=1,2$ as a function of the degeneracy lifting parameter $\xi$. For $\xi<0$, the state with $l=1$ is the ground state, for $\xi>0$ the $l=2$ state has lower energy. Right panel: overlap matrix elements of the levels $l=1,2$ to the ground states of $N \pm 1$ particles. (a) $\left\langle N-1 g\left|\Sigma_{\sigma} d_{j_{\alpha} \sigma}\right| N l=1\right\rangle$, (b) $\left\langle N-1 g\left|\Sigma_{\sigma} d_{j_{\alpha} \sigma}\right| N l=2\right\rangle$, (c) $\left\langle N l=1\left|\Sigma_{\sigma} d_{j_{\alpha} \sigma}\right| N+1 g\right\rangle$, and (d) $\left\langle N l=2\left|\Sigma_{\sigma} d_{j_{\alpha} \sigma}\right| N+1 g\right\rangle$.

$$
\frac{\left\langle N l\left|d_{j_{R} \sigma}\right| N+1 g\right\rangle\left\langle N+1 g\left|d_{j_{L} \sigma^{\prime}}^{\dagger}\right| N l^{\prime}\right\rangle}{E_{N l^{\prime}}-E_{N+1 g}}
$$

or

$$
\frac{\left\langle N l\left|d_{j_{L} \sigma}^{\dagger}\right| N-1 g\right\rangle\left\langle N-1 g\left|d_{j_{R} \sigma^{\prime}}\right| N l^{\prime}\right\rangle}{E_{N-1 g}-E_{N l^{\prime}}}
$$

with $|N \pm 1 g\rangle$ being the ground states with $N \pm 1$ electrons, are dominant in Eq. (5). The excited states contribute as well but less due to the energy difference in the denominator and their influence will not change the qualitative behavior of the differential conductance. It is therefore necessary to analyze separately the matrix elements $\left\langle N l\left|\Sigma_{\sigma} d_{j_{\alpha} \sigma}\right| N+1 g\right\rangle$ and $\left\langle N-1 g\left|\sum_{\sigma} d_{j_{\alpha} \sigma}\right| N l^{\prime}\right\rangle$. These are compared in Fig. 10, where we also investigate the behavior of the quasidegenerate levels as a function of the degeneracy lifting $\xi$. Plotting the energies of the two levels with $l=1,2$ versus $\xi$, we see that for $\xi<0$, the state with $l=1$ is the ground state, but for $\xi$ $>0$ the state with $l=2$ has lower energy. The overall dependence of the matrix elements on $\xi$ is rather weak, but the coupling to the ground states with $N \pm 1$ electrons of these two states is seen to be very different. The matrix element of the $N-1$ ground state with $l=1$ is about twice as large as the one with $l=2$, while for the elements with the $N+1$ ground state, this situation is reversed. This is reason why by changing the gate voltage one can tune the system into a configuration where $\gamma_{|2\rangle\langle 2|}^{R L}$ by far exceeds $\gamma_{|1\rangle\langle 1|}^{R L}$ such that the conductance increases monotonously even after the inelastic cotunneling threshold.

The cuts in Fig. 9 were done for $\xi<0$. Choosing instead $\xi>0$, the picture would be reversed and the conductance at lowest gate voltages (close to the side of the $N-1$ diamond) would be monotonously increasing while the conductance close to the $N+1$ diamond would now show the cusped line shape.

\section{B. Benzene}

We find exactly the same effect for a singly charged $(N$ $=7$ ) benzene molecule coupled to the leads in metaconfiguration. The spectrum of benzene exhibits a lot of degeneracies due to the $D_{6 h}$ symmetry of the molecule. ${ }^{29}$ The environment of a molecular junction can break the perfect symmetry of the molecule in various ways. ${ }^{30}$ As for the triple dot, we model this by ascribing a different on-site energy to the contact sites. We diagonalize $H_{\mathrm{QD}}$ exactly, and use the eigenstates and eigenvalues for each charge state to calculate all relevant cotunneling rates. The energy spectrum is shown in Fig. 11 and we now focus on the inelastic cotunneling corresponding to the weakly broken degeneracy in the $N=7$

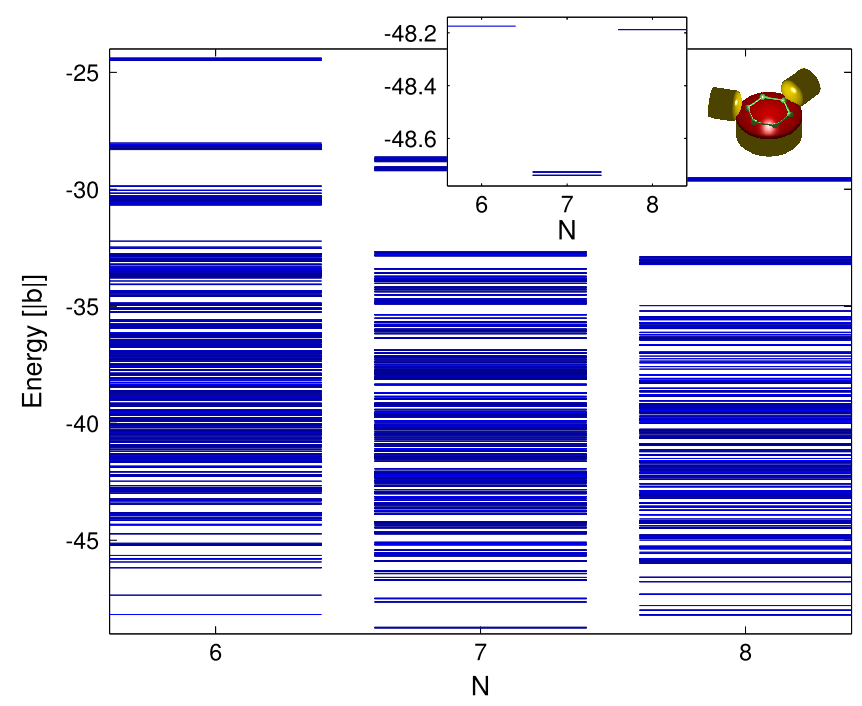

FIG. 11. (Color online) Spectrum of the benzene molecule for $N=6$ (neutral molecule), $N=7$ and $N=8$ electrons. The initially degenerate $N=7$ ground state is split due to the coupling to the leads (see inset). Parameters are $U=4|b|, V=2.4|b|$, and $\xi=-0.1|b|$. 


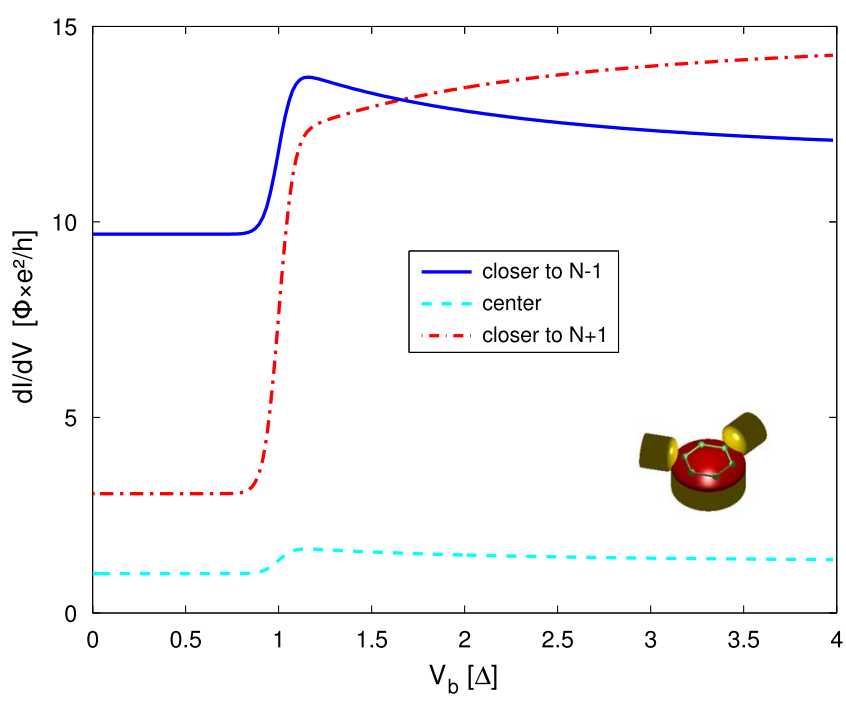

FIG. 12. (Color online) Cotunneling conductance vs bias voltage for a benzene molecule with $N=7$ electrons coupled to the leads in metaconfiguration. The three curves correspond to three values of the gate voltage being closer to the $N=6$ (neutral) particle diamond, at the center of the $N=7$ diamond and closer to the $N=8$ diamond. Parameters are $U=4|b|, V=2.4|b|$ (we consider nearest neighbor interaction only), $\xi=-0.1|b|$, and the remaining as in Fig. 8 .

state. In Fig. 12, we show three cuts through the $N=7$ Coulomb diamond of benzene at different gate voltages, one corresponding to the center and two toward the charge degeneracy points with $N=6$ and $N=8$. Also here, the line shape at the inelastic cotunneling threshold has a marked dependence on the gate voltage arising from a pronounced gate-voltage asymmetry in the cotunneling amplitudes. Closer to the $N$ $=6$ diamond, virtual tunneling-out processes are closer to resonance (have a smaller energy denominator) and closer to the $N=8$ diamond virtual tunneling-in processes dominate. As for the TD, the monotonously increasing conductance closer to the $N=8$ diamond is clearly seen to also have a larger step height.

\section{CONCLUSIONS}

In conclusion, we investigated cotunneling phenomena in complex quantum-dot systems and demonstrated that systems with weakly broken degeneracies can exhibit a marked gate-voltage dependence of the nonlinear cotunneling conductance traces. The effect relies on the nonequilibrium population of the excited state and is therefore most pro- nounced in devices coupled symmetrically to source and drain electrodes. The inelastic cotunneling threshold was found to be modulated so as to become either cusped or monotonously increasing, depending on whether the strongest transport channel is via the ground state or via the first excited state.

In Ref. 7, the inelastic cotunneling thresholds were shown to acquire a gate dependence due to the difference in tunneling-induced level shifts for the two different levels involved. Whereas that effect shows up with only tunnel coupling to a single lead, it is important to recognize that the gate-dependent modulation of the step which we discuss here relies entirely on the coupling to two different leads. Entering the Kondo regime for which such level shifts become important, both effects could be observed simultaneously and therefore it would be interesting to study this stronger coupled regime more closely in future studies.

From the technical point of view, we have demonstrated that the widely used simplification of the $T$-matrix approach (AprxI) is indeed in quantitative agreement with the exact fourth order perturbation theory (GME) in regions of gate and bias voltage for which sequential tunneling resonances are strongly suppressed. With a poorer separation of energy scales, i.e., when the inelastic cotunneling threshold is no longer much smaller than the charging energy, AprxI is insufficient but approximation AprxII and the $T$-matrix approach still perform very well and have a fairly large range of validity, within which they yield good agreement with the GME results. In particular, they describe very well the line shape of the inelastic cotunneling conductance for systems with weakly broken degeneracies, i.e., with $\Delta \ll E_{C}$. AprxI gives rise to substantial simplifications and allows writing occupation numbers and current in closed analytic form, despite the potential complexity of the underlying quantum-dot systems which is now wrapped up in the virtual transitionamplitudes comprising the effective exchange-cotunneling matrix elements, see Eq. (14). As such, AprxI can also be used for the investigation of Kondo-enhanced inelastic cotunneling $^{6}$ in more complex quantum dot or singlemolecule systems, where the most relevant terms in higher order perturbation theory will be the log-singular terms underlying the Kondo effect.

\section{ACKNOWLEDGMENTS}

We thank Andrea Donarini for fruitful discussions. We acknowledge financial support by the DFG within the research programs SPP 1243 and SFB 689.
${ }^{1}$ H. van Houten, C. W. J. Beenakker, and A. A. M. Staring, Single Charge Tunneling: Coulomb-Blockade Phenomena in Nanostructures (Plenum Press and NATO Scientific Affairs Division, New York, 1992).

${ }^{2}$ R. Schleser, T. Ihn, E. Ruh, K. Ensslin, M. Tews, D. Pfannkuche, D. C. Driscoll, and A. C. Gossard, Phys. Rev. Lett. 94, 206805
(2005).

${ }^{3}$ S. De Franceschi, S. Sasaki, J. M. Elzerman, W. G. van der Wiel, S. Tarucha, and L. P. Kouwenhoven, Phys. Rev. Lett. 86, 878 (2001).

${ }^{4}$ S. Sapmaz, P. Jarillo-Herrero, J. Kong, C. Dekker, L. P. Kouwenhoven, and H. S. J. van der Zant, Phys. Rev. B 71, 153402 
(2005).

${ }^{5}$ B. Babić, T. Kontos, and C. Schönenberger, Phys. Rev. B 70, 235419 (2004).

${ }^{6}$ J. Paaske, A. Rosch, P. Wölfle, N. Mason, C. M. Marcus, and J. Nygård, Nat. Phys. 2, 460 (2006).

${ }^{7}$ J. V. Holm, H. I. Jørgensen, K. Grove-Rasmussen, J. Paaske, K. Flensberg, and P. E. Lindelof, Phys. Rev. B 77, 161406(R) (2008).

${ }^{8}$ J. J. Parks, A. R. Champagne, G. R. Hutchison, S. Flores-Torres, H. D. Abruña, and D. C. Ralph, Phys. Rev. Lett. 99, 026601 (2007).

${ }^{9}$ E. A. Osorio, K. O’Neill, M. Wegewijs, N. Stuhr-Hansen, J. Paaske, T. Bjørnholm, and H. S. J. van der Zant, Nano Lett. 7, 3336 (2007).

${ }^{10}$ E. A. Osorio, T. Bjørnhom, J.-M. Lehn, M. Ruben, and H. S. J. van der Zant, J. Phys.: Condens. Matter 20, 374121 (2008).

${ }^{11}$ E. A. Osorio, K. Moth-Poulsen, H. S. J. van der Zant, J. Paaske, P. Hedegård, K. Flensberg, J. Bendix, and T. Bjørnholm, Nano Lett. 10, 105 (2010).

${ }^{12}$ N. Roch, S. Florens, V. Bouchiat, W. Wernsdorfer, and F. Balestro, Nature (London) 453, 633 (2008).

${ }^{13}$ V. N. Golovach and D. Loss, Phys. Rev. B 69, 245327 (2004).

${ }^{14} \mathrm{M}$. Wegewijs and Yu. Nazarov, arXiv:cond-mat/0103579 (unpublished).

${ }^{15}$ O. Parcollet and C. Hooley, Phys. Rev. B 66, 085315 (2002).

${ }^{16}$ J. Paaske, A. Rosch, and P. Wölfle, Phys. Rev. B 69, 155330 (2004).

${ }^{17}$ K. Kaasbjerg and K. Flensberg, Nano Lett. 8, 3809 (2008).

${ }^{18}$ H. Schoeller and G. Schön, Phys. Rev. B 50, 18436 (1994).

${ }^{19}$ J. König, J. Schmid, H. Schoeller, and G. Schön, Phys. Rev. B 54, 16820 (1996).

${ }^{20}$ J. König, H. Schoeller, and G. Schön, Phys. Rev. Lett. 76, 1715
(1996).

${ }^{21}$ M. Leijnse and M. R. Wegewijs, Phys. Rev. B 78, 235424 (2008).

${ }^{22} \mathrm{H}$. Bruus and K. Flensberg, Many-Body Quantum Theory in Condensed Matter Physics (Oxford University Press, Oxford, 2004).

${ }^{23}$ L. P. Kouwenhoven, D. G. Austing, and S. Tarucha, Rep. Prog. Phys. 64, 701 (2001).

${ }^{24}$ A. Bezryadin, A. R. M. Verschueren, S. J. Tans, and C. Dekker, Phys. Rev. Lett. 80, 4036 (1998)

${ }^{25}$ M. A. Reed, C. Zhou, C. J. Muller, T. P. Burgin, and J. M. Tour, Science 278, 252 (1997).

${ }^{26}$ R. Pariser and R. G. Parr, J. Chem. Phys. 21, 466 (1953); 21, 767 (1953).

${ }^{27}$ J. A. Pople, Farad. Trans. 49, 1375 (1953).

${ }^{28}$ M. H. Hettler, W. Wenzel, M. R. Wegewijs, and H. Schoeller, Phys. Rev. Lett. 90, 076805 (2003).

${ }^{29}$ G. Begemann, D. Darau, A. Donarini, and M. Grifoni, Phys. Rev. B 77, 201406 (2008).

${ }^{30}$ D. Darau, G. Begemann, A. Donarini, and M. Grifoni, Phys. Rev. B 79, 235404 (2009).

${ }^{31}$ V. Koerting, J. Paaske, and P. Wölfle, Phys. Rev. B 77, 165122 (2008).

${ }^{32}$ J. Koch, F. von Oppen, and A. V. Andreev, Phys. Rev. B 74, 205438 (2006).

${ }^{33}$ D. V. Averin, Physica B 194-196, 979 (1994).

${ }^{34}$ M. Turek and K. A. Matveev, Phys. Rev. B 65, 115332 (2002).

${ }^{35}$ C. Timm, Phys. Rev. B 77, 195416 (2008).

${ }^{36}$ S. Koller, M. Grifoni, M. Leijnse, and M. R. Wegewijs (unpublished).

${ }^{37}$ A. C. Hewson, The Kondo Problem to Heavy Fermions (Cambridge University Press, Cambridge, 1993). 